\title{
Asociación de niveles de lípidos y haplogrupos Amerindios de DNA mitocondrial en individuos chilenos hipercolesterolémicos tratados con Atorvastatina

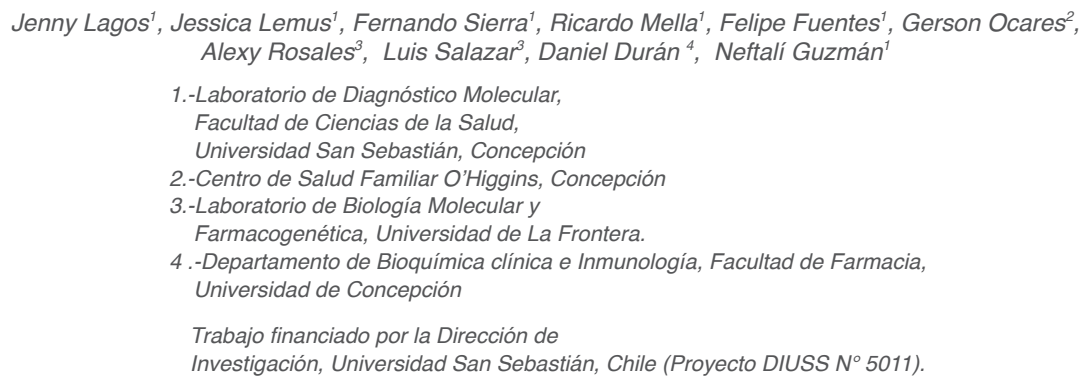

\section{Resumen:}

Introducción: La respuesta terapéutica a estatinas se ve influenciada por factores como la edad, género y etnicidad. Con respecto a esto, el background genético de la población chilena es predominantemente Amerindio, definido por la presencia de haplogrupos Amerindios $\mathrm{A}, \mathrm{B}, \mathrm{C}$ y D de DNA mitocondrial (mtDNA). Así, el objetivo del estudio fue evaluar la potencial asociación entre la presencia de haplogrupos Amerindios de mtDNA y niveles de lípidos en individuos chilenos hipercolesterolémicos tratados con Atorvastatina.

Métodos: Un total de 42 individuos en dos centros de salud del sur de Chile fueron incluidos en el estudio. En el grupo de pacientes se evaluó la presencia de haplogrupos Amerindios de mtDNA por PCR-RFLP, además de la cuantificación de Colesterol Total, Tri- glicéridos, Colesterol-HDL y Colesterol-LDL, antes y después del tratamiento con Atorvastatina (10 mg/día). Resultados: El $88.1 \%$ de los sujetos presentó algún haplogrupo Amerindio, no observándose diferencias en los niveles de lípidos pre- tratamiento de acuerdo al haplogrupo. Interesantemente, individuos de haplogrupo B presentaron niveles mayores de Colesterol Total (B: $254 \pm 30 \mathrm{mg} / \mathrm{dl} \mathrm{v} / \mathrm{s}$ C: $213 \pm 48 \mathrm{mg} / \mathrm{dl}$, D: $230 \pm 50 \mathrm{mg} / \mathrm{dl} ; \mathrm{p}=0.0319)$ y Colesterol-LDL (B: 157 $\pm 34 \mathrm{mg} / \mathrm{dl} \mathrm{v} / \mathrm{s} \mathrm{C}: 118 \pm 45 \mathrm{mg} / \mathrm{dl}, \mathrm{D}: 135 \pm 42 \mathrm{mg} / \mathrm{dl}$; $\mathrm{p}=0.0344)$ post-tratamiento.

Conclusiones: El haplogrupo B se asocia a niveles mayores de lípidos post-tratamiento en pacientes tratados con Atorvastatina. Estos hallazgos sugieren por primera vez, que la presencia de haplogrupo B de mtDNA determinaría una menor respuesta al tratamiento con Atorvastatina en individuos chilenos con background genético amerindio.

\section{Association of serum lipid levels and mitochondrial DNA Amerindian haplogroups in hypercholesterolemic subjects receiving atorvastatin}

Background: Therapeutic response to statins is influenced by age, gender and ethnicity. The genetic background of the Chilean population is predominantly Amerindian, defined by the presence of mitochondrial DNA (mtDNA) Amerindian haplogroups A, B, C and D Aim: to evaluate a possible association of mtDNA
Amerindian haplogroups and serum lipid levels in hypercholesterolemic Chilean subjects receiving atorvastatin

Methods: 42 subjects from southern Chile were included. The presence of mtDNA Amerindian haplo groups was evaluated by PCR-RFLP; in addition, total 
Asociaci $n$ de niveles de I pidos y haplogrupos Amerindios de DNA mitocondrial en individuos chilenos hipercolesterol micos... Lagos J, et al.

cholesterol, triglycerides, HDL-cholesterol and LDLcholesterol were measured before and after treatment with atorvastatin $10 \mathrm{mg} /$ day.

Results: $88.1 \%$ of subjects exhibited some Amerindian haplogroup. No relation of lipid levels with haplogroups was observed before treatment. Interestingly, haplogroup B individuals had higher levels of total cholesterol compared to other haplogroups after treatment (haplogroup B : $254 \pm 30 \mathrm{mg} / \mathrm{dl} ; \mathrm{C}: 213 \pm$ $48 \mathrm{mg} / \mathrm{dl}$; D : $230 \pm 50 \mathrm{mg} / \mathrm{dl}, \mathrm{p}=0.0319)$. Corresponding levels for LDL-cholesterol after treatment in the

\section{Introducción:}

Diversos estudios han demostrado la efectividad clínica de las estatinas, avalando su utilización en pacientes que presentan niveles elevados de colesterol. Este fármaco contribuye a reducir significativamente la morbimortalidad cardiovascular, ya que además de su efecto hipolipemiante posee múltiples acciones ateroprotectoras $^{1-4}$. Sin embargo, la evidencia científica muestra una importante variabilidad en la respuesta terapéutica, lo que sería consecuencia de factores biológicos y fisiológicos del paciente ${ }^{5,6}$.

La variabilidad observada podría explicarse en parte por la presencia de polimorfismos en genes candidatos que influyen sobre la farmacocinética y farmacodinámica de este medicamento. Dentro de este grupo, se incluyen genes codificantes para proteínas que actúan en la absorción intestinal de colesterol, que regulan la producción o la homeostasis intracelular de colesterol, factores de transcripción y enzimas que regulan el metabolismo de las estatinas ${ }^{7-15}$. Sin embargo, los marcadores moleculares descritos muestran importantes diferencias en diversas poblaciones, lo que podría ser explicado por la influencia del carácter étnico de las poblaciones estudiadas ${ }^{16}$.

Estudios de genética poblacional han demostrado que la población chilena presenta un componente mayoritariamente amerindio ${ }^{17}$. La caracterización de una población amerindia puede ser realizada a través de marcadores del genoma mitocondrial, siendo posible identificar cuatro haplogrupos denominados A, B, C y D. Estos haplogrupos son definidos por un marcador específico: la ganancia de un sitio de restricción para la enzima Hae III en la posición 663 para el haplogrupo A, la deleción de 9pb en la región intergénica COII/tRNALys para el haplogrupo $\mathrm{B}$, una pérdida de un sitio de restricción para la enzima Hinc II en la posición 13.259 para el haplogrupo $\mathrm{C}$ y la pérdida de un sitio de restricción para la enzima Alu I en la posición 5176 para el haplogrupo $\mathrm{D}^{18-20}$. three groups were $157 \pm 34,118 \pm 45$ and $135 \pm 42 \mathrm{mg} /$ $\mathrm{dl}$, respectively, $\mathrm{p}=0.0344$.

Conclusion: Compared to other haplogroups, haplogroup B is associated to higher levels of lipids after treatment with atorvastatin. For the first time, these findings suggest that the presence of mtDNA haplogroup B determines a dimished response to atorvastatin in Chilean subjets with an Amerindian genetic background.

Keywords: mtDNA haplogroups, hypercholesterolemia, atorvastatin

Considerando que Atorvastatina es la estatina más utilizada en Chile y que no existen antecedentes de estudios que evalúen la asociación de la etnicidad con respuesta al tratamiento en población chilena, el objetivo de este estudio fue evaluar la asociación de haplogrupos Amerindios de mtDNA con niveles de lípidos en individuos chilenos hipercolesterolémicos tratados con Atorvastatina.

\section{Métodos:}

\section{Sujetos de estudio}

Se estudiaron 42 individuos, de ambos sexos, con diagnóstico de hipercolesterolemia y pertenecientes al programa de salud cardiovascular de dos centros de salud familiar de la región del Bío-Bío y de la Araucanía. Todos fueron sometidos a tratamiento con Atorvastatina de 10 $\mathrm{mg}$ /día, durante cuatro semanas y se les evaluó el perfil lipídico completo antes y después de este período de tratamiento. Fueron excluidos todos aquellos sujetos que presentaron enfermedad hepática, renal, hipotiroidismo, terapia concomitante con fibratos y tratamiento con Atorvastatina como medida preventiva secundaria, sin presentar diagnóstico de hipercolesterolemia. Datos demográficos e historia de hipertensión y diabetes mellitus fueron evaluados para cada individuo.

Todos los individuos incluidos aprobaron su participación en el estudio mediante la firma de un consentimiento informado, de acuerdo a los criterios descritos en la declaración de Helsinki.

\section{Determinaciones bioquímicas}

Para las determinaciones bioquímicas se recolectaron muestras de sangre venosa posterior a un ayuno de 12 horas en tubos sin anticoagulante. Los sueros obtenidos se almacenaron a $-20^{\circ} \mathrm{C}$ hasta el momento de su determinación. Se evaluaron niveles de Colesterol Total, Triglicéridos, Colesterol HDL y LDL, este último calculado mediante fórmula de Friedewald. 


\section{Genotipificación de haplogrupos amerindios en $m+D N A$}

Para el análisis de haplogrupos amerindios en mtDNA se realizó la extracción de ácidos nucleicos a partir de sangre total anticoagulada con EDTA-K3 (1mg/ml). La obtención de ADN se realizó a partir de concentrado leucocitario de acuerdo al método descrito por Salazar et al $(1998)^{21}$.

La genotipificación de haplogrupos amerindios de mtDNA se realizó de acuerdo a metodología previamente descrita ${ }^{17}$. Las reacciones de amplificación fueron realizadas en un volumen final de $25 \mu \mathrm{l}$ conteniendo $50 \mathrm{ng}$ de DNA genómico, $100 \mathrm{nM}$ de cada partidor, $200 \mathrm{mM}$ de cada dNTP, 1 unidad de Taq DNA polimerasa y buffer de PCR $(\mathrm{KCl} 50 \mathrm{mM}, 2 \mathrm{mM} \mathrm{MgCl} 2,20$ $\mathrm{mM}$ (NH4)2SO4, 75 mM Tris-HCl, pH 9.0).

Para determinar la presencia o ausencia de los tres sitios de restricción polimórficos, característicos de los haplogrupos A, C y D de población amerindia, se realizó la digestión con endonucleasas específicas HaeIII, HincII y AluI, respectivamente. Finalmente, los fragmentos de restricción fueron evaluados por electroforesis en un gel de agarosa al 3\% teñido con bromuro de etidio. Con respecto al marcador que define al haplogrupo B y que consiste en una deleción de una de las dos repeticiones de 9 pb ubicadas en la región intergénica $\mathrm{V}$, la visualización se realizó por medio de una electroforesis en gel de poliacrilamida al 10\%, directamente de los productos de PCR obtenidos.

\section{Análisis estadístico}

Las frecuencias de haplogrupos Amerindios de mtDNA fueron estimadas directamente. Para el análisis de asociación entre niveles de lípidos y presencia de algún Haplogrupo Amerindio de mtDNA, se comparó cada haplogrupo con todos los otros incluidos en un mismo grupo, asumiendo significancia estadística con un valor de $\mathrm{p}<0.05$.

\section{Resultados:}

Las características clínicas y demográficas de los pacientes incluidos en el estudio se presentan en la Tabla 1. Por su parte, la frecuencia de haplogrupos amerindios de mtDNA en la población estudiada se presenta en la Tabla 2. Es destacable que de la población de pacientes, el $88.1 \%$ presentó algún haplogrupo Amerindio de mtDNA, observándose una mayor frecuencia de haplogrupo B.

Los niveles de lípidos de acuerdo a haplogrupos de mtDNA se muestran en la Tabla 3. En relación a los niveles de lípidos pre-tratamiento, no se observó una asociación significativa con algún haplogrupo. Interesantemente, al analizar los niveles de lípidos posttratamiento, se observó asociación significativa con el haplogrupo B, por lo que individuos de este haplogrupo presentaron niveles mayores de colesterol total y colesterol LDL respecto de individuos portadores de los otros haplogrupos analizados.

\begin{tabular}{|c|c|c|}
\hline & Hombres $(n=8)$ & Mujeres $(n=34)$ \\
\hline $\begin{array}{l}\text { Edad, a os } \\
\text { (Media } \pm \text { DS) }\end{array}$ & $61 \pm 13$ & $58 \pm 15$ \\
\hline Diabetes mellitus & $0(0 \%)$ & $3(8.8 \%)$ \\
\hline Hipertensi $n$ & $3(37.5 \%)$ & 19 (55.8\%) \\
\hline
\end{tabular}

Tabla 1. DS=Desviación Standard;

\begin{tabular}{|c|c|c|}
\hline Haplogrupos & $\mathbf{n}$ & $\%$ \\
\hline A & 3 & 7 \\
\hline B & 14 & 33 \\
\hline C & 10 & 24 \\
\hline D & 10 & 24 \\
\hline NA & 5 & 12 \\
\hline Total & 42 & 100 \\
\hline
\end{tabular}

Tabla 2. NA= No Amerindios 


\section{Tabla 3. Niveles de lípidos de acuerdo a haplogrupos amerindios de mtDNA} en pacientes hipercolesterolémicos en tratamiento con Atorvastatina

\begin{tabular}{|c|c|c|c|c|}
\hline Lípidos & $A(n=3)$ & $B(n=14)$ & $C(n=10)$ & $D(n=10)$ \\
\hline \multicolumn{5}{|l|}{ Pre-tratamiento } \\
\hline Colesterol Total & $260 \pm 28$ & $276 \pm 15$ & $270 \pm 30$ & $277 \pm 25$ \\
\hline Triglic ridos & $173 \pm 50$ & $196 \pm 49$ & $212 \pm 71$ & $206 \pm 62$ \\
\hline Col-HDL & $50 \pm 9.1$ & $58 \pm 10$ & $55 \pm 13$ & $57 \pm 13$ \\
\hline Col-LDL & $175 \pm 29.7$ & $179 \pm 17.3$ & $172 \pm 27.1$ & $179 \pm 26.9$ \\
\hline \multicolumn{5}{|c|}{ Post-tratamiento } \\
\hline Colesterol Total & $210 \pm 49$ & $254 \pm 29.7^{a}$ & $213 \pm 47.9$ & $230 \pm 50.4$ \\
\hline Triglic ridos & $214 \pm 23$ & $197 \pm 50$ & $205 \pm 78$ & $202 \pm 44$ \\
\hline Col-HDL & $48 \pm 3.8$ & $59 \pm 8.7$ & $54 \pm 10.8$ & $57.4 \pm 9.8$ \\
\hline Col-LDL & $119 \pm 49.5$ & $157 \pm 34.2^{a}$ & $118 \pm 45.3$ & $135 \pm 42.6$ \\
\hline
\end{tabular}

Tabla 3. $a=p<0.05$

\section{Discusión:}

La Atorvastatina constituye uno de los fármacos más utilizados en el tratamiento de la hipercolesterolemia. Sin embargo, la evidencia disponible muestra una importante variabilidad en la respuesta terapéutica, influenciada por factores como la edad, género y etnicidad ${ }^{5,6}$.

En el presente estudio, se evaluó la potencial asociación entre la presencia de haplogrupos Amerindios de mtDNA y niveles de lípidos en individuos chilenos hipercolesterolémicos tratados con Atorvastatina. Los resultados muestran que el componente Amerindio es predominante en la población incluida en el estudio, lo que se correlaciona con otros antecedentes que muestran la elevada frecuencia de haplogrupos amerindios de mtDNA en Chile ${ }^{22-23}$. Además, la evidencia disponible muestra que la distribución de haplogrupos mitocondriales en población general no es homogénea en Chile, observándose una frecuencia elevada de haplogrupos A y B en el Norte chileno, mientras que los haplogrupos $\mathrm{C}$ y $\mathrm{D}$ aumentarían progresivamente hacia el sur del país ${ }^{17,22,23}$. Destaca la mayor frecuencia de haplogrupo B en la población de pacientes hipercolesterolémicos, que contrasta con lo observado en población general donde se observa la tendencia descrita en la literatura (datos no mostrados). Este hallazgo muestra una tendencia que sugeriría una potencial asociación entre el haplogrupo B e hipercolesterolemia, lo que sería necesario evaluar en un mayor número de individuos, no existiendo estudios previos que evalúen esta posible asociación en población con ancestros amerindios.

Al comparar los niveles plasmáticos de lípidos de acuerdo a haplogrupos de mtDNA, no se observa asociación significativa entre la presencia de alguno de los haplogrupos analizados y las concentraciones de lípidos pre-tratamiento. En este sentido, la evidencia científica que asocia la presencia de haplogrupos de mtDNA con niveles de lípidos séricos es escasa. Kokaze et al. (2001) ${ }^{27}$, en una población japonesa de 461 sujetos aparentemente sanos, demostraron que individuos varones portadores del polimorfismo mtDNA A5178C presentaban concentraciones mayores de colesterol LDL, mientras que mujeres portadoras mostraban niveles mayores de triglicéridos. Además, Dahmani et al. $(2008)^{28}$, describieron que en deportistas, la presencia de haplogrupos caucásicos HV se asociaría a niveles elevados de colesterol LDL, mientras que la presencia de haplogrupo JT determinaría concentraciones menores esta lipoproteína. $\mathrm{Al}$ analizar los niveles de lípidos post-tratamiento, se observa que individuos de haplogrupo B presentan una mayor concentración de colesterol total y colesterol LDL, siendo este último parámetro un reconocido factor de riesgo cardiovascular. Este hallazgo permitiría sugerir que la presencia de un determinado haplogrupo que determine niveles elevados de colesterol LDL podría definir una mayor susceptibilidad a eventos cardiovasculares en individuos portadores de este marcador del genoma mitocondrial.

De acuerdo a lo anterior, diversos autores sugieren que la presencia de variantes en el genoma mitocondrial podría 
modificar la susceptibilidad a enfermedades complejas, entre ellas las enfermedades cardiovasculares. Recientemente, Castro et al. describieron en población caucásica, una asociación entre la presencia de haplogrupo $\mathrm{T}$ y cardiomiopatía hipertrófica ${ }^{24}$. En contraste, estudios en otros grupos poblacionales muestran que algunos haplogrupos de mtDNA ejercerían un efecto protector frente a Infarto cerebro vascular isquémico e Infarto al Miocardio ${ }^{25,26}$. Sin embargo, hasta ahora no existe evidencia con respecto a la potencial influencia que tendría la presencia de haplogrupos Amerindios de mtDNA sobre la susceptibilidad a enfermedades crónicas no transmisibles en poblaciones con background genético predominantemente amerindio, como la mayoría de las poblaciones latinoamericanas.

En cuanto a la asociación de niveles elevados de lípidos post-tratamiento con la presencia de un haplogrupo amerindio de mtDNA y el impacto que esto tendría sobre la respuesta terapéutica a estatinas, la mayoría de los estu- dios farmacogenómicos han intentado explicar la variabilidad en la respuesta a este fármaco basándose en la búsqueda de polimorfismos en genes nucleares que afecten tanto la farmacocinética como la farmacodinámica del medicamento. Nuestros hallazgos constituyen el primer antecedente internacional, de asociación entre un marcador genético de etnicidad presente en el genoma mitocondrial y niveles mayores de lípidos en pacientes tratados con Atorvastatina, lo que podría interpretarse como una menor respuesta a la acción del fármaco.

En resumen, los resultados muestran que la presencia del haplogrupo B de mtDNA se asocia a niveles mayores de colesterol total y colesterol LDL en pacientes hipercolesterolémicos chilenos, pudiéndose constituir en un biomarcador de menor respuesta al tratamiento con Atorvastatina, lo que debería ser evaluado en un mayor número de individuos y en otros grupos poblacionales latinoamericanos.

\section{Referencias:}

1. MENNICKENT S, BRAVO M, CALVO C, AVELLO M, Efectos pleiotrópicos de las estatinas. Rev Méd Chile, 2008; 136: 775-782.

2. CALVO C. Manejo farmacológico de las dislipidemias en la prevención de las enfermedades cardiovasculares. An R Acad Nac Farm 2004; 70: 417-425.

3. BUCHER H, GRIFFICH L, GUYATT G. Systematic review on the risk and benefit of different cholesterol lowering interventions. Arteroscler Thromb Vasc Biol 1999; 19: 187-195.

4. VAUGHAN CJ, GOTTO AM, BASSON CT. The evolving role of statins in the management of atherosclerosis. J Am Coll Cardiol 2000; 35: 1-10

5. KAJINAMI K, TAKEKOSHI $\mathrm{N}$, BROUSSEAU ME, SCHAEFER EJ. Pharmacogenetics of HMG-CoA reductase inhibitors: exploring the potential for genotype-based individualization of coronary heart disease management. Atherosclerosis 2004; 177:219-34.

6. SCHMITZ G, LANGMANN T. Pharmacogenomics of cholesterol-lowering therapy. Atherosclerosis 2006; 44: 75-89

7. KAJINAMI K, BROUSSEAU ME, NARTSUPHA C, ORDOVAS JM, SCHAEFER EJ. ATP binding cassette transporter G5 y G8 genotypes and plasma lipoprotein levels before and after treatment with Atorvastatin. J Lipid Res. 2004; 45: 653-656.

8. HAGBERG J, WILUND K, FERRELL R. Apo E gene and gene-environment effects on plasma lipoprotein-lipid levels. Phisiol Genom 2000; 4: 101-108.

9. MAITLAND-VAN DER ZEE A.H, STRICKER B.H.C, KLUNGEL O.H, MANTEL-TEEUWISSE A.K, KASTELEIN J.J. Adherence to na dosing of beta-hidroxy-beta-methylglutaril coenzyme a reductase inhibitors in the general population differs according to apolipoprotein E-genotypes. Pharmacogenetics 2003; 13: 219-223.

10. CHASMAN D, POSADA D, SUBRAHMANYAN L, COOK N, STANTON V, RIDKER P. Pharmacogenetic Study of Statin Therapy and Cholesterol Reduction. JAMA 2004; 291: 2821-2827.

11. DUAN X, ZHU W, LI Y, ZHAO Y, DAO J, XIAO Y. The effect of sterol regulatory element-binding protein 2 polymorphism on the serum lipid in northern Chines subjects. J Lipid Res 2005; 46: 252-257.

12. CHAVES F.J, REAL J, GARCIA-GARCIA A.B, CIVERA M, ARMENGOD M.E, ASCASO J.F. Genetics Diagnosis of Familial Hypercholesterolemia in a South European Outbreed Population: Influence of Low-density Lipoprotein (LDL) receptor Gene Mutations on Treatment Response to Simvastatin in total, LDL and HDL-density Lipoprotein Cholesterol. J Clin Endocrinol Metabol 2001; 86: 4926-4932

13. SPOSITO A.C, GONBERT S, BRUCKERT E, ATASSI M, BEUCLER I, AMSELLEN S. Magnitude of HDL cholesterol variation after high-dose atorvastatin in genetically determined at the LDL receptor locus in patients with 
homozygous familial hypercholesterolemia. Arterioscler. Thromb Vasc Biol 2003; 23: 2078-2082.

14. KAJINAMI K, BROUSSEAU ME, ORDOVAS JM, SCHAEFER EJ. CYP3A4 genotypes and plasma lipoprotein levels before and after treatment with Atorvastatin in primary hypercholesterolemia. Am J Cardiol 2004; 93: 104-107.

15. WILLRICH M, HIRATA M, GENVIGIR F, ARAZI S, REBECCHI I, RODRIGUES A. CYP3A5*3A Allele is associated with reduced lowering -lipid response to atorvastatin in individuals with hipercholesterolemia. Clin Chim Acta 2008; 398: 15-20.

16. FERDINAND K. Ethnic, Gender, and Age-related Differences in the Treatment of Dyslipidemia. The Am J Man Care 2006; 12: s400-S404.

17. MORAGA M, ROCCO P, MIQUEL JF. Mitochondrial DNA polymorphisms in Chilean aboriginal population: implications for the peopling of the southern cone of the continent. Am J Phys Anthropol 2000; 113: 19-29.

18. SANTOS S, RIBEIRO-DOS SANTOS A, MEYER D, ZAGO M. Multiple founder haplotypes of mitochondrial DNA Amerindians revealed by RFLP and sequency. Ann Hum Genet 1996; 60: 305-319.

19. BAILLIET G, ROTHAMMER F, CARNESE F, BRAVI C, BIANCHI N. Founder mitochondrial haplotypes in Amerindian populations. Am J Hum Genet 1994; 54: 27-33.

20. GARCIA F, MORAGA M, VERA S, HENRIQUEZ H, LLOP E. mtDNA microevolution in Southern Chile's archipielagos. Am J Phys Anthropol 2006; 129: 473-481.

21. SALAZAR L.A., HIRATA M, CAVALLI S, MACHADO MO, HIRATA RD. Optimized procedure DNA isolation from fresh and cryopreserved clotted human blood useful in clinical molecular testing. Clin Chem 1998; 44: 174850.

22. ROCCO P, MORALES C, MORAGA M, MIQUEL J, NERVI F, LLOP E. Composición genética de la población chilena: Distribución de polimorfismos de DNA mitocondrial en grupos originarios y en la población mixta de Santiago. Rev Méd Chil 2002; 130 : 125-31

23. HENRÍQUEZ H, MORAGA M, LLOP E, ROTHHAMMER E. Caracterización genético molecular de habitantes de caleta Paposo último reducto Chango en Chile. Rev Méd Chile 2004; 132: 663-672.

24. CASTRO MG, HUERTA C, REGUERO JR, SOTO MI, DOMÉNECH E, ALVAREZ V. Mitochondrial DNA haplogroups in Spanish patients with hypertrophic cardiomyopathy. Int J Cardiol 2006; 112: 202-206.

25. ROSA A, FONSECA B, KRUG T, MANSO H, GOUVEIA L, ALBERGARIA I. Mitochondrial haplogroup H1 is protective for ischemic stroke in Portuguese patients. BMC Med Genet 2008, 9: 57-67.

26. NISHIGAKI Y, YAMADA Y, FUKU N, MATSUO H, SEGAWA T, WATANABE S. Mitochondrial haplogroup N9b is protective against myocardial infarction in Japanese males. Hum Genet 2007; 120: 827-836.

27. KOKAZE A, ISHIKAWA M, MATSUNAGA N, YOSHIDA M, SEKINE Y, TERUYA K. Association of the mitochondrial DNA $5178 \mathrm{~A} / \mathrm{C}$ polymorphism with serum lipid levels in the Japanese population. Hum Genet 2001; 109, 521-525.

28. DAHMANI Y, MARCUELLO A, DÍEZ-SANCHEZ C, RUIZ-PESINI E, MONTOYA J, LÓPEZ-PÉREZ M. Association of human mitochondrial DNA variants with plasma LDL levels. Mitochondrion 2008; 8: 247-253. 